\title{
Débits respiratoires et activités quotidiennes : paramètres de l'exposition aux substances inhalées*
}

\author{
M. ROY ${ }^{\star \star}$, J.L. MALARBET ${ }^{\star \star}$, C. COURTAY ${ }^{\star \star}$
}

(Manuscrit reçu le 15 janvier 1993)

RÉSUMÉ Pour connaître la quantité de substances toxiques Inhalées, on fait appel a des valeurs standard du volume d'alr respiré quotldiennement par les personnes exposées aux gaz et aux aérosols. A l'occasion de la révision du modèle dosimétrique des voles respiratoires de la Commission Internationale de protection radiologique (CIPR), ces valeurs ont été actualisées pour l'adulte et complétées pour l'enfant, grâce à des données récentes sur la ventilation au repos et à l'exercice et à l'aide d'enquêtes sur l'emploi du temps des individus types de la population publiées par I'Institut nationat de la statistique et des études économiques (INSEE). Les résultats sont donnés sous forme de tableaux pour les adultes et les enfants de 3 mols, 1, 5, 10 et 15 ans ; ils sont légèrement différents de ceux publiés auparavant par la CIPR et par le Comité scientifique des Nations unles pour l'étude des effets des rayonnements ionisants (UNSCEAR).

ABSTRACT The intake of inhaled toxic substances is based upon the air volumes breathed every day by people under exposure to gases and aerosols. On the occasion of the revision of the respiratory tract model by the International commission on radlological protection (ICRP), modern standards have been assessed for average inspired air volumes according to age and sex. Recent data of breathing rates as a function of physical activity have been recorded, and economical surveys recently published by the National institute of statistics and economical studies (INSEE) provided time budgets and activitles of specific categories of the population. The results were calculated for adults and children, 3 months, 1, 5, 10 and 15 years old. These values are slightly different from those formerly published by ICRP and the United Nations scientific committee on the effects of atomic radiation (UNSCEAR).

\section{Introduction}

L'évaluation des quantités de substances inhalées est importante pour les toxiques industriels transportés par l'air sous forme de gaz ou d'aérosols ainsi que pour l'exposition naturelle au radon des travailleurs et du

* Communication "poster" présentée au Congrès IRPA'B, Montréal, 17-22 mai 1992.

* Commissariat à l'énergie atomique, Institut de protection et de sûreté nucléaire, DPHD, SDOS, BP 6, 92265 Fontenay-aux-Roses Cedex.

Service de médecine du travail de la Hague, BP 270, 50107 Cherbourg. 
public. Elle nécessite la connaissance de paramètres individuels influençant l'exposition tels que l'âge, l'activité physique et l'emploi du temps quotidien, tenant compte du temps passé en plein air ou à l'intérieur des habitations et des lieux de travail. Des valeurs avaient déjà été publiées par la CIPR $[18,19]$ et I'UNSCEAR $[25,26]$. Les plus récentes utilisaient les données de Brown [2] basées sur une étude conduite en 1978 par la British broadcasting corporation (BBC) sur l'emploi du temps des personnes âgées de plus de 5 ans en Grande-Bretagne. D'autres données moins détaillées avaient été précédemment recueillies sur environ 30000 personnes adultes dans 12 pays d'Europe, aux Etats-Unis, au Pérou et dans I'ancienne Union Soviétique [21]. En 1985, un rapport statistique sur les facteurs d'exposition a été publié par l'Environmental protection agency américaine (EPA) [1]. La révision du modèle dosimétrique pulmonaire de la CIPR a donné l'occasion de revoir et de compléter ces paramètres à l'aide de données détaillées de la physiologie de la ventilation tenant compte de l'activité physique et de l'âge. Des enquêtes récentes de l'INSEE ont fourni d'autre part des emplois du temps très précis sur différentes catégories de la population. La combinaison de ces deux types d'information nous a permis de calculer des volumes moyens d'air inhalés quotidiennement par les individus dans les différents environnements.

\section{Rethodes}

\subsection{Emplois du temps}

Les enquêtes de l'INSEE ont chiffré la durée des activités de plusieurs groupes de personnes représentatives des principales composantes de la population française : employé sédentaire homme et femme, femme au foyer avec ou sans enfant, étudiant [13, 15-17]. En éliminant deux periodes de 15 jours, l'une durant les fêtes de fin d'année, l'autre durant les vacances d'été, 16047 personnes interrogées ont décrit leurs journées de 5 en 5 minutes ; le total devait comprendre un nombre équivalent de chacun des jours de la semaine. Chaque emploi du temps n'est donc pas représentatif de l'individu qui l'a décrit, mais de l'ensemble des emplois du temps de grands groupes d'individus. On a établi des distributions statistiques de ces différentes composantes de la population. On en a extrait les fractiles pour y étudier également de manière statistique les durées d'activité en choisissant la médiane $(50 \%$ des individus interrogés) comme paramètre représentatif.

Les données concernant les enfants sont disponibles dans ces enquêtes seulement à partir de 15 ans $[12,14]$. Pour les enfants d'âge scolaire de 4 à 14 ans, nous avons utilisé le guide de la scolarité [22].

Pour les enfants de moins de 3 ans, nous avons extrapolé à partir de l'emploi du temps de leur mère. 
Nous avons aussi utilisé pour tous les âges le rapport publié par Médiamétrie [11] sur l'audience télévisuelle. A partir de toutes ces informations, lieux de séjour et activités ont été répartis de façon condensée en 3 environnements principaux :

- intérieur de l'habitation : sommeil, temps physiologique (toilette, repas, etc.), travail ménager (nettoyage, cuisine, soins aux enfants, bricolage), travail scolaire à la maison, etc., temps libre (lecture, télévision, etc.) ;

- intérieur d'un autre lieu : travail sédentaire (bureau, atelier, école, etc.), autres activités (associations, spectacles, etc.) ;

- extérieur : voyages (comprenant le trajet au travail ou à l'école), bricolage et courses, sports et promenades.

Ces valeurs, ainsi que les fractions de $24 \mathrm{~h}$ passées dans chacun de ces environnements, sont présentées dans le tableau I pour les adultes et dans le tableau II pour les enfants (ce sont des moyennes établies sur toute l'année, à l'exclusion des deux périodes de vacances indiquées dans la méthodologie).

Dans ces études, il manquait les travailleurs en plein air (agriculture, bâtiment, travaux publics, ...) et les mineurs ; nous leur avons alors attribué les mêmes horaires de travail que les hommes employés sédentaires, mais avec un niveau plus élevé d'activité physique (travail dur).

\subsection{Paramètres de ventilation.}

En fonction du niveau d'exercice, la fréquence respiratoire $\left(f_{R}\right)$, le volume courant $\left(V_{T}\right)$ et la ventilation par minute $\left(\dot{V}_{E}\right)$ ont été fixés pour l'homme adulte selon 4 activités types : sommeil, repos assis, exercice léger (travail de laboratoire, d'atelier, de nettoyage, etc.), exercice dur ne pouvant dépasser $2 \mathrm{~h}$ par jour (mines, bâtiment, agriculture, entraînement sportif, etc., [5, 20, 23]. Tab. III). Chez les femmes et les enfants, ces valeurs ont été adaptées proportionnellement à celles des hommes d'après des données, obtenues expérimentalement par des méthodes standard et sur des séries suffisantes de cycles ventilatoires pour permettre un calcul de la moyenne individuelle avec une déviation standard inférieure à $10 \%$. Elles sont, le plus souvent, exprimées en fonction du poids corporel moyen pour les âges considérés $V_{T}=10 \mathrm{ml} . \mathrm{kg}^{-1}$ à partir de 5 ans)

On a ainsi sélectionné pour les sujets de :

- 3 mois : $V_{T}$, d'après Gaultier [7] : sommeil : $6 \mathrm{ml}^{\circ} \mathrm{kg}^{-1}$, exercice léger : $10 \mathrm{ml}^{\mathrm{kg}}{ }^{-1}$,

$f_{\mathrm{R}}$, d'après Gaultier [7] et Taussig et al. [24]

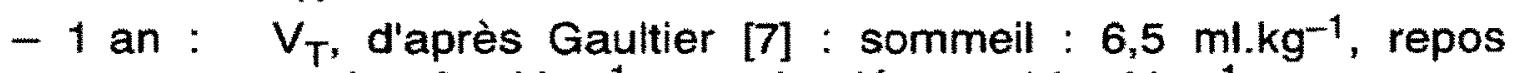
assis : $9 \mathrm{ml} . \mathrm{kg}^{-1}$, exercice léger : $11 \mathrm{ml} \mathrm{kg}^{-1}$;

$f_{\mathbf{R}}$, d'après Gaultier [7] et Taussig et al. [24]. 
TABLEAU

Emplol du temps des adultes

Adults" time budget

\begin{tabular}{|c|c|c|c|c|}
\hline . & $\begin{array}{l}\text { Femme } \\
\text { aves } \\
\text { plusteurs } \\
\text { entants }\end{array}$ & $\begin{array}{l}\text { Femme } \\
\text { sans } \\
\text { enfant }\end{array}$ & $\begin{array}{l}\text { Homme } \\
\text { employe }\end{array}$ & $\begin{array}{l}\text { Femme } \\
\text { employes }\end{array}$ \\
\hline \multirow{2}{*}{\multicolumn{5}{|c|}{$\begin{array}{l}\text { Mabitation } \\
\text { Sommeil }\end{array}$}} \\
\hline & & & & \\
\hline Temps physiologiq & $2 \mathrm{n} 51$ & $3 h 30$ & 2 ค 55 & 2 h 47 \\
\hline Travail a la maison & $8 \mathrm{ho0}$ & $6 \mathrm{~h} 20$ & $2 \mathrm{ho0}$ & $4 h 54$ \\
\hline $\begin{array}{l}\text { Temps libre } \\
\text { (T.V.) }\end{array}$ & $\begin{array}{l}2 h 34 \\
(2 h 30)\end{array}$ & $\begin{array}{l}3 \text { h } 05 \\
(2 h 30)\end{array}$ & 2 ho7 & $1 \mathrm{~h} 41$ \\
\hline Total & 22 \& 11 & $22 \cap 05$ & $15 h 27$ & $17 h 51$ \\
\hline Autres battiments & & & & \\
\hline Travail & - & - & 5 h 32 & 3 h 52 \\
\hline Autres & $O \perp 32$ & Oh 35 & $1 \ln 00$ & 0 h 32 \\
\hline Total & $O H 32$ & 0735 & $6 h 32$ & $4 h 24$ \\
\hline Exténleur & & 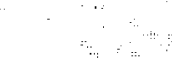 & $\cdots$ & 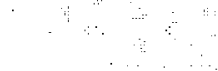 \\
\hline Courses, bricolage & Oh 44 & $0 h 50$ & $0 h 41$ & on 30 \\
\hline Voyage & $0 \mathrm{h12}$ & - & $1 h 00$ & $1 \hbar 00$ \\
\hline Jeux, sports & o h 21 & $0 \mathrm{~h} 30$ & o h 20 & on 15 \\
\hline Total & $1 \% 17$ & $\operatorname{th} 20$ & $2 h 01$ & $\ln 45$ \\
\hline Habitation & $92,5 \%$ & $92,0 \%$ & $64,5 \%$ & $74,5 \%$ \\
\hline Autres bâtiments & $2,0 \%$ & $2,5 \%$ & $27,5 \%$ & $18,5 \%$ \\
\hline Extérieur. & $5,5 \%$ & $5,5 \%$ & $8,0 \%$ & $7,0 \%$ \\
\hline
\end{tabular}

\% Pourcentage de la journée.

- 5 ans : $V_{T}$ et $f_{\mathrm{R}}$ : sommeil et repos assis, d'après Taussig et al. [24], exercice léger, d'après Godfrey et al. [10].

- 10 et 15 ans : $V_{\mathrm{T}}$ et $f_{\mathrm{R}}$ : sommeil, d'après Gaultier et al. [8], repos assis, d'après Gaultier et al. [8] et Zapletal [27], exercices léger et dur, d'après Godfrey [9], Godfrey et al. [10], Cooper et Weiler-Ravell [3] et Flandrois et al. [6]. 
TABLEAU II

Emploi du temps des enfants Children's time budget

\begin{tabular}{|c|c|c|}
\hline \multicolumn{3}{|l|}{ Habitation } \\
\hline Sommeil & $12 \mathrm{~h} 00$ & $10 \mathrm{~h} 00$ \\
\hline Temps physiologique & $3 \mathrm{~h} 00$ & $3 \mathrm{~h} 00$ \\
\hline Etude (travail à la maison) & - & $1 \mathrm{~h} 40$ \\
\hline Jeux, temps libre & $2 h 50$ & $3 \cap 00$ \\
\hline $19 h 00$ & $17 h 50$ & 17 h 40 \\
\hline \multicolumn{3}{|l|}{ Autres bâtiments } \\
\hline Ecole ou nurserie & $3 \mathrm{n} 00$ & $3 \mathrm{~h} \mathrm{00}$ \\
\hline Jeux & - & o h 10 \\
\hline $4 h 00$ & $3 h 00$ & $3 \circ 10$ \\
\hline Extérieur & & \\
\hline Voyage & $0 \mathrm{~h} 45$ & oh 45 \\
\hline Récréation & $1 \mathrm{~h} 45$ & $1 \mathrm{~h} 25$ \\
\hline Jeux, sports & & \\
\hline $1 \mathrm{hoo}$ & $3 h 10$ & $3 \circ 10$ \\
\hline Habitation & $74,5 \%$ & $74 \%$ \\
\hline Autres bâtiments & $12,5 \%$ & $13 \%$ \\
\hline Extérieur & $13 \%$ & $13 \%$ \\
\hline
\end{tabular}

$\%$ pourcentage de la journée.

- Adultes : $V_{\mathrm{T}}$ et $f_{\mathrm{R}}$, sommeil et repos assis, d'après Cotes [4], exercices léger et dur, d'après Cooper et Weiler-Ravell [3].

Ces paramètres sont détaillés dans le tableau IV. 
TABLEAU III

Débits respiratoires de l'homme adulte au cours des activités journalières Adult breathing rates during his dally activities

\begin{tabular}{|c|c|c|}
\hline $\begin{array}{l}\text { Activité type } \\
\text { a }\end{array}$ & $\begin{array}{l}\mathrm{Dob} \mathrm{bi} \\
\mathrm{min}\end{array}$ & Dóbit ventilatoire $\dot{V}_{E}$ \\
\hline Sommeil & 7,5 & 0,45 \\
\hline Repos assis & 9 & 0,54 \\
\hline Exercice léger & 25 & 1,5 \\
\hline Exercice dur & 50 & 3 \\
\hline
\end{tabular}

\subsection{Volumes d'air respirés quotidiennement}

Les activités, décrites dans les environnements définis ci-dessus, ont été associées aux débits de ventilation nécessaires à chacun selon âge et sexe.

- Dans la population générale et chez les travailleurs sédentaires, les 24 heures sont ainsi réparties :

- à l'intérieur (habitation, lieu de travail ou autres) entre le sommeil et l'éveil : 1/3 en repos assis, 2/3 en exercice léger.

- à l'extérieur (plein air, train, bus, voitures,...) en éveil : $1 / 2$ en repos assis, $3 / 8$ en exercice léger, 1/8 en exercice dur.

Chez les autres travailleurs (travail dur) à l'extérieur ou dans les mines, et sur leur lieu de travail seulement : $7 / 8$ en exercice léger, $1 / 8$ en exercice dur.

Les tableaux $V$ pour les adultes et $V I$ pour les enfants fournissent le détail quotidien de ces paramètres d'environnement, d'activités et de débits respiratoires qui leur sont associés. Les volumes d'air respirés sont totalisés pour chaque situation. Afin qu'ils soient valables pour toutes les personnes du public, n'importe quel jour de leur vie, ces valeurs sont données sous forme de moyennes sur les 7 jours d'une semaine normale ; ceci permettra, en cas de besoin, de revenir facilement à des circonstances définies pour un individu considéré plus particulièrement.

\section{Discussion}

Le temps passé par les adultes à l'intérieur des bâtiments est resté relativement proche de celui indiqué en 1981 par l'étude faite aux EtatsUnis et dans 12 pays d'Europe dont 6 villes de France [21]. II n'a pas changé pour les hommes employés $(22 \mathrm{~h})$ mais a légèrement diminué pour les femmes au foyer $(22,7$ contre $23,3 \mathrm{~h})$. Cependant, les chiffres de I'INSEE sont supérieurs de 5 à $10 \%$ à ceux qui ont été publiés par I'EPA [1]. 
DEBITS RESPIRATOIRES ET ACTIVITES QUOTIDIENNES

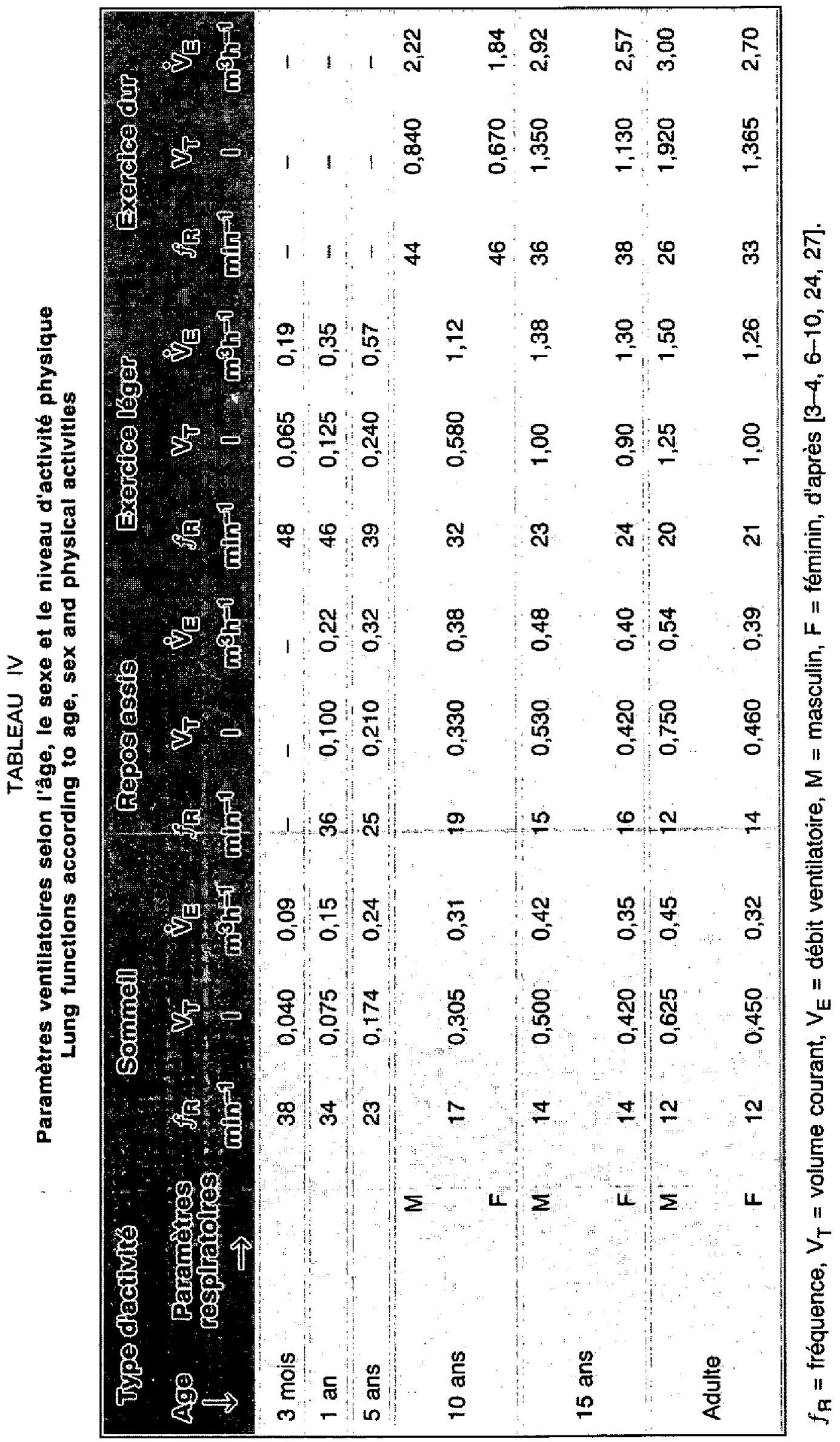




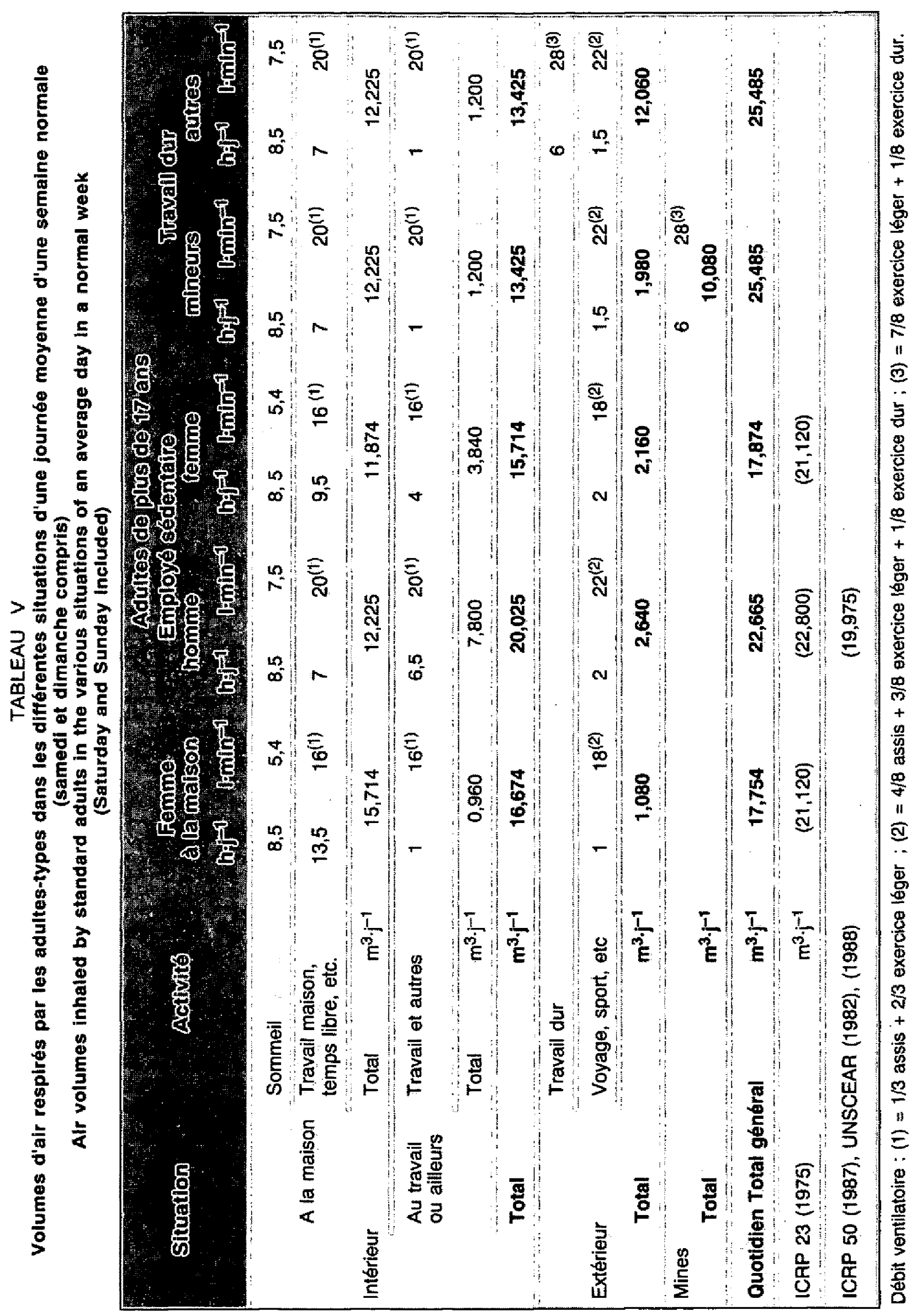


DÉBITS RESPIRATOIRES ET ACTIVITÉS QUOTIDIENNES

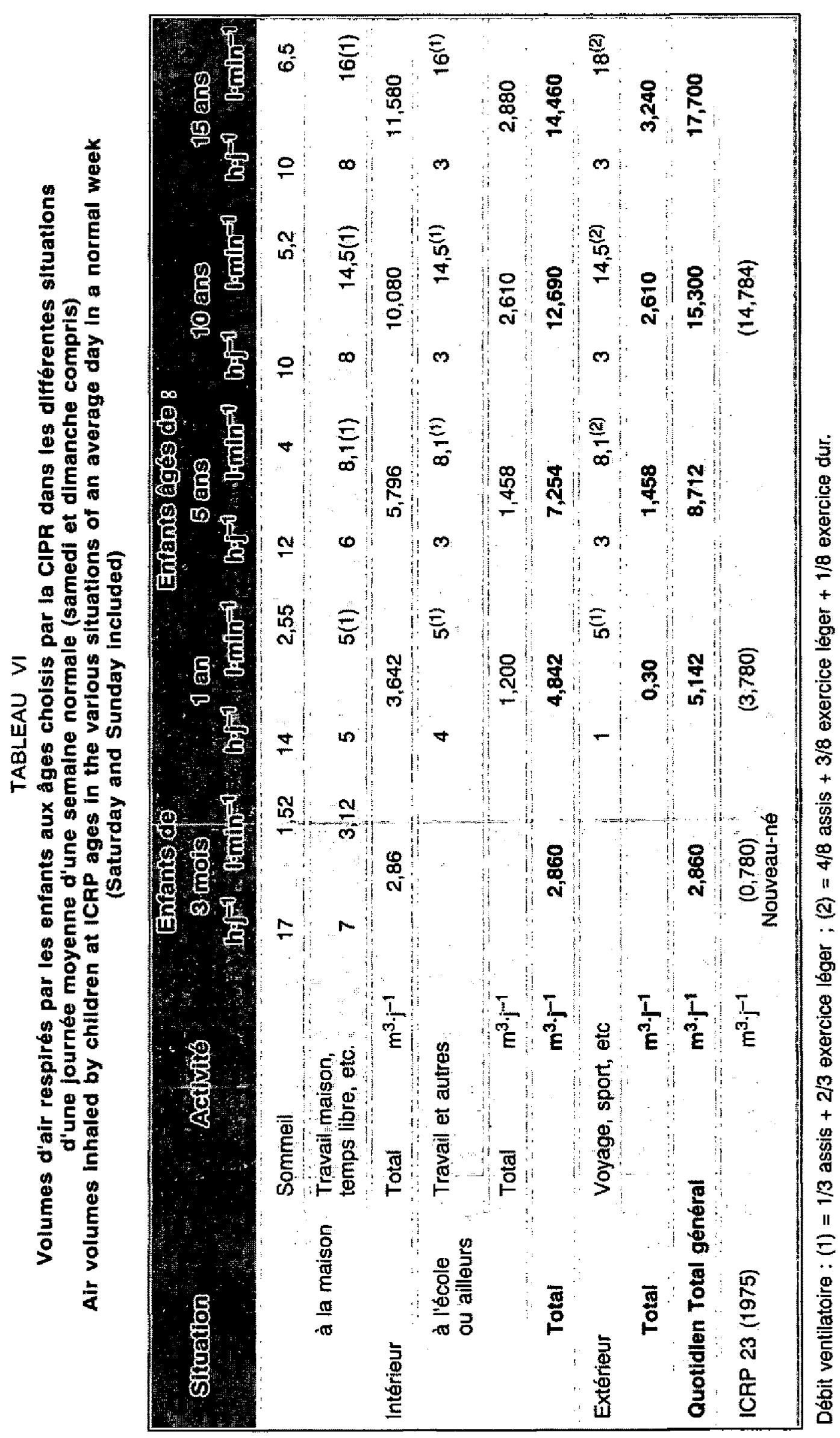

RADIOPROTECTION - VOL. 28 - No $3(1993)$ 
Pour les enfants, le temps passé à l'école semble plus court dans notre étude ( $3 \mathrm{~h}$ par jour sur toute la semaine) que dans celle de Brown [2] (7 h par jour pendant 5 jours par semaine). Cependant, il est probablement assez voisin, car on peut supposer que les $7 \mathrm{~h}$ quotidiennes d'école doivent être interrompues par des récréations à l'extérieur.

La publication 23 de la CIPR [18] recommandait la division de la journée en 3 périodes de $8 \mathrm{~h}$ pour l'homme adulte $8 \mathrm{~h}$ de sommeil avec une ventilation de 7,5 I. $\mathrm{min}^{-1}, 8 \mathrm{~h}$ de travail et $8 \mathrm{~h}$ d'autres activités avec une ventilation de $20\left(. \mathrm{min}^{-1}\right)$. Elle donnait aussi des valeurs pour la femme, le nouveau-né et les enfants de 1 et 10 ans. Ensuite, pour les hommes du public, I'UNSCEAR 82 [25] et la Publication 50 de la CIPR [19] ont recommandé les valeurs de $0,75 \mathrm{~m}^{3} \cdot \mathrm{h}^{-1}$ pendant $19 \mathrm{~h}$ à l'intérieur, et de $1 \mathrm{~m}^{3} \cdot \mathrm{h}^{-1}$ pendant $5 \mathrm{~h}$ à l'extérieur. Ces chiffres ont été simplifiés par I'UNSCEAR 88 [26] en fixant la ventilation à $0,8 \mathrm{~m}^{3} \cdot \mathrm{h}^{-1}$ pour tous les adultes et dans toutes les situations.

Pour les adultes, il y a peu d'écart entre les volumes moyens journaliers d'air respirés fournis ainsi par la CIPR et par I'UNSGEAR et ceux calculés par notre méthode. En revanche, pour l'enfant de moins de 10 ans, les volumes fixés par la Publication 23 sont environ la moitié de cèux que nous avons calculés.

\section{Conclusion}

Pour mettre en cuvre les recommandations de la CIPR concernant l'exposition, aussi bien des personnes du public que des travailleurs, aux substances inhalées, des paramètres détaillés de ventilation et d'emploi du temps ont été revus en détail à l'aide de données récentes. Les débits de ventilation varient en fonction du poids et donc de l'âge des individus et aussi avec leur activité physique ; on peut ainsi définir des valeurs standard. L'emploi du temps des différentes catégories de personnes de la population basé sur les enquêtes françaises semble utilisable pour d'autres pays occidentaux (Europe, USA...) jouissant d'un climat tempéré.

Par comparaison avec les volumes d'air respirés quotidiennement et calculés par cette méthode, les valeurs publiées auparavant par la CIPR étaient peu différentes pour l'adulte et l'enfant de 10 ans, mais deux fois plus faibles pour les enfants plus jeunes. Ceci conduisait à une sous-estimation de leur exposition aux radionucléides inhalés et de leur incorporation.

Remerciements : Ce travail a été financé en partie par la Commission des Communautés Européennes, DG12 : Contrat F 13 PCT920064A - Inhalation and ingestion of radionuclides. 


\section{RÉFÉRENCES}

[1] ANDERSON E., BRAUNE N., DUTLESKY S., RAMIG J., WARRI T. Development of statistical distributions of ranges of standard factors in exposure assessments (EPA 600/8.85/010). Washington D.C. : Environmental protection agency, 1985.

[2] BROWN L. - National radiation survey in the UK : indoor occupancy factors. Fadiat. Prot. Dosim., 1983, 5, 203-208.

[3] COOPER D.M., WEILER-RAVELL D. - Gas exchange response to exercise in children. Am. Rev. Respir. Dis.,1984, 129, Suppl. \$47-S48.

[4] COTES J.E. - Lung function assessment and application in medicine. Oxford: Blackwell, 1979.

[5] DEJOURS P. - Physiologie de la respiration. Paris : Flammarion Médecine Sciences, 1982.

[6] FLANDROIS R., GRANDMONTAGNE H., MAYET M.H., FAVIER R., FRUTOSO J. - La consommation d'oxygène maximale chez le jeune français, sa variation avec l'âge, le sexe et l'entraînement. J. Physiol., Paris, 1982, 78, 186-194.

[7] GAULTIER C. - Développement des volumes puimonaires pendant les 36 premiers mois de la vie chez l'homme. Thèse de doctorat en biologie humaine, $\mathrm{CHU}$ St-Antoine, Paris, 1978.

[8] GAULTIER C., PERRET L., BOULE N., BUVRY A., GIRARD F. - Occlusion pressure and breathing pattern in healthy children. Respir. Physiol., 1981, 46, 71-80.

[9] GODFREY S. - Exercise testing in children. Londres : Saunders, 1973.

[10] GODFREY S., DAVIES C.T.M., WOZNIAK E., BARNES C.A. - Cardio-respiratory response to exercise in normal children. Clin. Sci., 1971, 40, 419-431.

[11] INSTITUT MEDIAMETRIE. - Le livre de l'audience 1990. Paris : J. Aglietta, 1991.

[12] INSTITUT NATIONAL DE LA STATISTIQUE ET DES ETUDES ECONOMIQUES (INSEE). - Contours et caractères. Les jeunes de 15 à 24 ans. Paris : INSEE, 1988 .

[13] INSTITUT NATIONAL DE LA STATISTIQUE ET DES ETUDES ECONOMIQUES (INSEE). ARNAL N., DUMONTIER F., PAIRE R. - Les téléspectateurs. Econ. Stat., $1989, n^{\circ} 227$.

[14] INSTITUT NATIONAL DE LA STATISTIQUE ET DES ETUDES ECONOMIQUES, (INSEE). GALLAND O. - La vie quotidienne des jeunes du lycée au mariage. Econ. Stat., 1989, $n^{\circ} 223$.

[15] INSTITUT NATIONAL DE LA STATISTIQUE ET DES ETUDES ECONOMIQUES, (INSEE). GARRIGUES P. - Une France un peu plus sportive qu'il y a 20 ans, grâce aux femmes. Econ. Stat., 1989, $n^{\circ} 224$.

[16] INSTITUT NATIONAL DE LA STATISTIQUE ET DES ETUDES ECONOMIQUES, (INSEE). GRIMIER G. - Des journées les plus contraignantes aux journées les plus pénibles. Econ. Stat., 1989, $n^{\circ} 227$.

[17] INSTITUT NATIONAL DE LA STATISTIQUE ET DES ETUDES ECONOMIQUES, (INSEE). ROY C. - La gestion du temps des hommes et des femmes, des actifs et des inactifs. Econ. Stat., 1989, $n^{\circ} 223$.

[18] INTERNATIONAL COMMISSION ON RADIOLOGICAL PROTECTION (ICRP). - Reference man, anatomical, physiological and metabolic characteristics (ICRP Publication 23). Oxford : Pergamon press, 1975.

[19] INTERNATIONAL COMMISSION ON RADIOLOGICAL PROTECTION (ICRP). - Lung cancer risk from indoor exposures to radon daughters (ICRP Publication 50). Oxford : Pergamon press, 1987. 
[20] MONOD H., FLANDROIS R. - Physiologie du sport. Paris : Masson, 1985.

[21] MOSCHANDREAS D.J. - Exposure to pollutants and daily time budgets of people. Bull. N.Y. Acad. Med., 1981, 57 (10), 845-859.

[22] OFFICE NATIONAL DINFORMATION SUR LES ENSEIGNEMENTS ET LES PROFESSIONS (ONISEF) et MINISTERE DE L'EDUCATION NATIONALE. - Guide de la scolarité de la maternelle à l'université. Paris : Livre de poche, 1987.

[23] SCHERRER J. - Précis de physiologie du travail. Notions d'ergonomie. Paris : Masson, 1981.

[24] TAUSSIG L.M., HARRIS T.R., LEBOWITZ M.D. - Lung function in infants and young children. Am. Rev. Respir. Dis., 1977, 116, 233-239.

[25] UNITED NATIONS SCIENTIFIC COMMITTEE ON THE EFFECTS OF ATOMIC RADIATION (UNSCEAR). - lonizing radiations : sources and biological effects. (UN Publication E.82. IX.8) New York : United Nations, 1982.

[26] UNITED NATIONS SCIENTIFIC COMMITTEE ON THE EFFECTS OF ATOMIC RADIATION (UNSCEAR). - Sources, effects and risks of ionizing radiation. (UN Publication E.88. (X.7). New York: United Nations, 1988.

[27] ZAPLETAL A. - Lung function testing in children and adolescents $(H$. Herzog series). Bâle : Karger, 1987. 